Directeur de LA PUblication / Publication diRECTOR: Bruno David,

Président du Muséum national d'Histoire naturelle

RÉDACTEUR EN CHEF / EDITOR-IN-CHIEF: Jean-Philippe Siblet

ASSISTANTE DE RÉDACTION / ASSISTANT EDITOR: Sarah Figuet (naturae@mnhn.fr)

Mise EN PAGE / PAGE LAYOUT: Sarah Figuet

COMITÉ SCIENTIFIQUE / SCIENTIFIC BOARD:

Luc Abbadie (UPMC, Paris)

Luc Barbier (Parc naturel régional des caps et marais d'Opale, Colembert)

Aurélien Besnard (CEFE, Montpellier)

Vincent Boullet (Expert indépendant flore/végétation, Frugières-le-Pin)

Hervé Brustel (École d'ingénieurs de Purpan, Toulouse)

Patrick De Wever (MNHN, Paris)

Thierry Dutoit (UMR CNRS IMBE, Avignon)

Éric Feunteun (MNHN, Dinard)

Romain Garrouste (MNHN, Paris)

Grégoire Gautier (DRAAF Occitanie, Toulouse)

Olivier Gilg (Réserves naturelles de France, Dijon)

Frédéric Gosselin (Irstea, Nogent-sur-Vernisson)

Patrick Haffner (UMS PatriNat, Paris)

Frédéric Hendoux (MNHN, Paris)

Xavier Houard (OPIE, Guyancourt)

Isabelle Leviol (MNHN, Concarneau)

Francis Meunier (Conservatoire d'espaces naturels - Hauts-de-France, Amiens)

Serge Muller (MNHN, Paris)

Francis Olivereau (DREAL Centre, Orléans)

Laurent Poncet (UMS PatriNat, Paris)

Nicolas Poulet (OFB, Vincennes)

Jean-Philippe Siblet (UMS PatriNat, Paris)

Laurent Tillon (ONF, Paris)

Julien Touroult (UMS PatriNat, Paris)

COUVERTURE / COVER:

Site expérimental montrant la prairie réhabilitée et les enclos expérimentaux illustrant les placettes de transfert de foin et de gestion différenciée.

Crédit photo: Didier Alard.
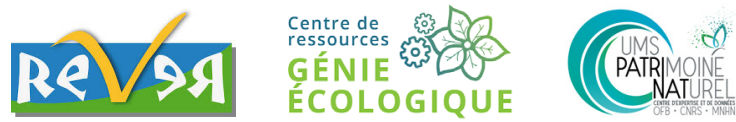

Naturae est une revue en flux continu publiée par les Publications scientifiques du Muséum, Paris

Naturae is a fast track journal published by the Museum Science Press, Paris

Les Publications scientifiques du Muséum publient aussi / The Museum Science Press also publish:

Adansonia, Zoosystema, Anthropozoologica, European Journal of Taxonomy, Geodiversitas, Cryptogamie sous-sections Algologie, Bryologie, Mycologie, Comptes Rendus Palevol.

Diffusion - Publications scientifiques Muséum national d'Histoire naturelle

CP $41-57$ rue Cuvier F-75231 Paris cedex 05 (France)

Tél.: 33 (0)140794805 / Fax: 33 (0)1 40793840

diff.pub@mnhn.fr / https://sciencepress.mnhn.fr

(c) Publications scientifiques du Muséum national d'Histoire naturelle, Paris, 2021

ISSN (électronique / electronic): 1638-9387 


\title{
Processus initiaux et dynamique de restauration écologique d'une prairie alluviale par transfert de foin et gestions différenciées
}

\author{
Myriam GARROUJ \\ Marie-Lise BENOT \\ Emmanuel CORCKET \\ Université Bordeaux, BIOGECO, INRAE, \\ Bât B2-CS 50023, F-33615 Pessac (France) \\ mgarrouj@gmail.com \\ marie-lise.benot@u-bordeaux.fr \\ emmanuel.corcket@imbe.fr \\ Lilian MARCHAND \\ SUEZ EAU France SAS - Le LyRE \\ 91 rue Paulin, CS 71706, F-33050 Bordeaux Cedex (France) \\ lilian.marchand@suez.com \\ Didier ALARD \\ Université Bordeaux, BIOGECO, INRAE, \\ Bât B2-CS 50023, F-33615 Pessac (France) \\ didier.alard@u-bordeaux.fr
}

Soumis le 8 avril 2020 | Accepté le 8 octobre 2020 | Publié le 22 septembre 2021

MOTS CLÉS

Communauté de référence,

diagramme TERNAIRE,

fauche,

pâturage,

vallée de la Garonne.

\begin{abstract}
Garrouj M., Benot M.-L., Corcket E., Marchand L. \& Alard D. 2021. - Processus initiaux et dynamique de restauration
écologique d'une prairie alluviale par transfert de foin et gestions différenciées, in Gourdain P. (éd.), REVER 10 - 10ème Colloque du Réseau d'Échange et de Valorisation en Écologie de la Restauration, Paris, 19-21 mars 2019. Naturae 2021 (20): 277-291. https://doi.org/10.5852/naturae2021a20

RÉSUMÉ

Nous étudions expérimentalement les effets de la gestion initiale sur les premiers stades de restauration d'une prairie alluviale à partir d'une ancienne culture de maïs, dans le sud-ouest de la France. Une expérimentation par blocs randomisés a été définie pour tester les effets du transfert de foin associé au hersage superficiel du sol et de la gestion par pâturage appliquée, soit immédiatement, soit plusieurs mois après le transfert de foin, ou par fauche appliquée annuellement en début ou en fin d'été. La richesse en espèces était toujours supérieure dans les placettes ayant reçu du foin, par rapport aux placettes témoin. L'origine, la direction et l'amplitude de la trajectoire de chaque placette étaient dépendantes du type de gestion appliquée. Le pâturage initial par les brebis, appliqué en même temps que le transfert de foin, a augmenté le recrutement des espèces de la communauté de référence, par un effet de contrôle de la compétition des espèces pré-existantes et le maintien d'une fenêtre d'opportunité pour le recrutement de ces espèces suffisamment longue. Le type de gestion appliqué dès le transfert conditionne l'origine de la trajectoire de restauration, tandis que sa direction et son amplitude sont liées à la gestion sur le moyen terme. À partir de la cinquième année d'expérimentation, ce sont des événements stochastiques liés à l'environnement ou la démographie des espèces qui semblent donner l'avantage aux contingences locales (banque de graines et/ou pluie de graines) aboutissant à une déviation de la trajectoire de restauration. Nous montrons néanmoins que le pâturage initial, très tôt dans le processus de restauration, semble approprié pour accélérer le recrutement des espèces de l'écosystème de référence.
\end{abstract}


KEY WORDS

Reference plant community,

TERNAIRE diagram, mowing, grazing trajectory,

Garonne river valley.

\begin{abstract}
Early-stage processes implied in the installation of a plant community and representation of restoration dynamic of alluvial grassland after hay transfer and different managements.

We study experimentally the effects of early stage management on restoration trajectories of a flood-plain grassland from a previously arable land in southwestern France. A randomized block design experiment was performed to test the coupling effects of hay transfer and soil harrowing in association with different management treatments: grazing immediately or several months after hay transfer, or either early or late summer mowing. Species richness was significantly higher in hay inoculated than in control plots. The origin, direction, and magnitude of the trajectory of hay inoculated plots all depended on the kind of management applied. Sheep grazing applied at the same time as hay transfer enhanced the recruitment of reference species as from the first experimental year, because it controlled aboveground competition and maintained the window of opportunity for species recruitment open for a sufficiently longer period of time. Our findings show that the kind of management applied simultaneously to harrowing and hay transfer influences the origin of a grassland trajectory, while its direction and magnitude are dependent on the management applied in subsequent years. On a longer time scale (five years), environmental or demographic stochasticity may deflect restoration trajectories towards more contingent assemblages, linked to seed rain or seed bank characterising the site. Grazing immediately after hay transfer may, however, be appropriate to accelerate the recruitment of species from the reference grassland.
\end{abstract}

\section{INTRODUCTION}

La plupart des grandes vallées alluviales européennes ont connu une perte massive d'habitats naturels et semi-naturels du fait de diverses actions anthropiques telles que la chenalisation, l'endiguement, l'agriculture ou l'urbanisation qui ont affecté leurs régimes hydrologiques (Bayley 1995). La perte des habitats primaires, liés aux régimes de perturbation des fleuves, est corrélée à l'extension de l'agriculture des derniers siècles et aux endiguements successifs (Sparks et al. 1990) qui ont transformé les paysages fluviaux en mosaïques agricoles plus ou moins intensives (Alard et al. 2002). Les habitats semi-naturels secondaires qui en ont résulté, principalement au sein de paysages bocagers et herbagers, constituent néanmoins des zones humides alluviales d'un fort intérêt patrimonial et fonctionnel, hébergeant une biodiversité riche et originale (Barnaud 1998). Au cours des dernières décennies, ces habitats secondaires liés à une agriculture traditionnelle et considérés comme prioritaires dans le réseau Natura 2000, ont également fortement régressé suite à l'intensification de l'agriculture, entraînant la mise en culture généralisée et la simplification des paysages, avec pour conséquence une perte considérable de la biodiversité et des services écologiques associés à ces plaines alluviales comme par exemple la pollinisation, la régulation des ravageurs ou l'atténuation des crues (Tscharntke et al. 2005; Henle et al. 2008). Dans ce contexte, les vallées des grandes rivières et fleuves ont fait l'objet de nombreuses opérations de restauration écologique (Poudevigne et al. 2002; Dufour \& Piégay 2009) visant à rétablir les habitats primaires inféodés aux régimes fluviaux (e.g., annexes hydrauliques, Paillex et al. 2009) ou, lorsque c'était impossible, des habitats secondaires de prairies semi-naturelles (Mchergui et al. 2014).

La restauration de prairies semi-naturelles à partir d'anciennes terres cultivées a fait l'objet d'études variées vis-àvis des méthodes utilisées, en particulier dans les plaines alluviales (Muller et al. 1998; Woodcock et al. 2011). D'importantes limites à la restauration sont communes aux opérations menées dans les zones humides en général (Moreno-Mateos et al. 2012). Les facteurs limitant la qualité des opérations de restauration écologique, qui ont principalement été visés via des approches expérimentales, concernent les conditions pédologiques des sites (Mchergui et al. 2014) et le filtre de dispersion des graines, particulièrement crucial dans un contexte de fragmentation des paysages (Pywell et al. 2002; Fattorini \& Halle 2004). Pour remédier à cette contrainte majeure de l'arrivée des graines jusqu'au site restauré, les méthodes du transfert de graines ou de foin ont été largement appliquées dans les expériences de restauration (Engst et al. 2016; Scotton 2016). Il reste malgré tout des questions peu explorées concernant les effets quantitatifs (amplitude) ou qualitatifs (direction) du transfert initial de foin et l'importance de la gestion initiale appliquée sur le site après transfert pour expliquer l'amplitude et la direction des trajectoires temporelles de restauration des communautés végétales (Pywell et al. 2011). Le transfert de foin initial accélère-t-il une trajectoire de restauration par ailleurs largement déterminée par la gestion à moyen terme ou a-t-il une influence majeure et permanente sur cette trajectoire (Young et al. 2005) ? La gestion précoce après restauration, agissant en particulier via le régime de perturbation (pâturage, fauche) sur l'accumulation de biomasse et les interactions biotiques au sein du tapis végétal, a-t-elle un rôle sur l'établissement des plantules des espèces transférées et par quel processus (Török et al. 2011) ? Nous proposons de répondre à ces questions via une approche expérimentale associant transfert de foin et gestion différenciée des premiers stades de restauration au sein d'une plaine alluviale anciennement cultivée (maïsiculture) et reconvertie en prairie permanente. Deux hypothèses sont particulièrement testées: 


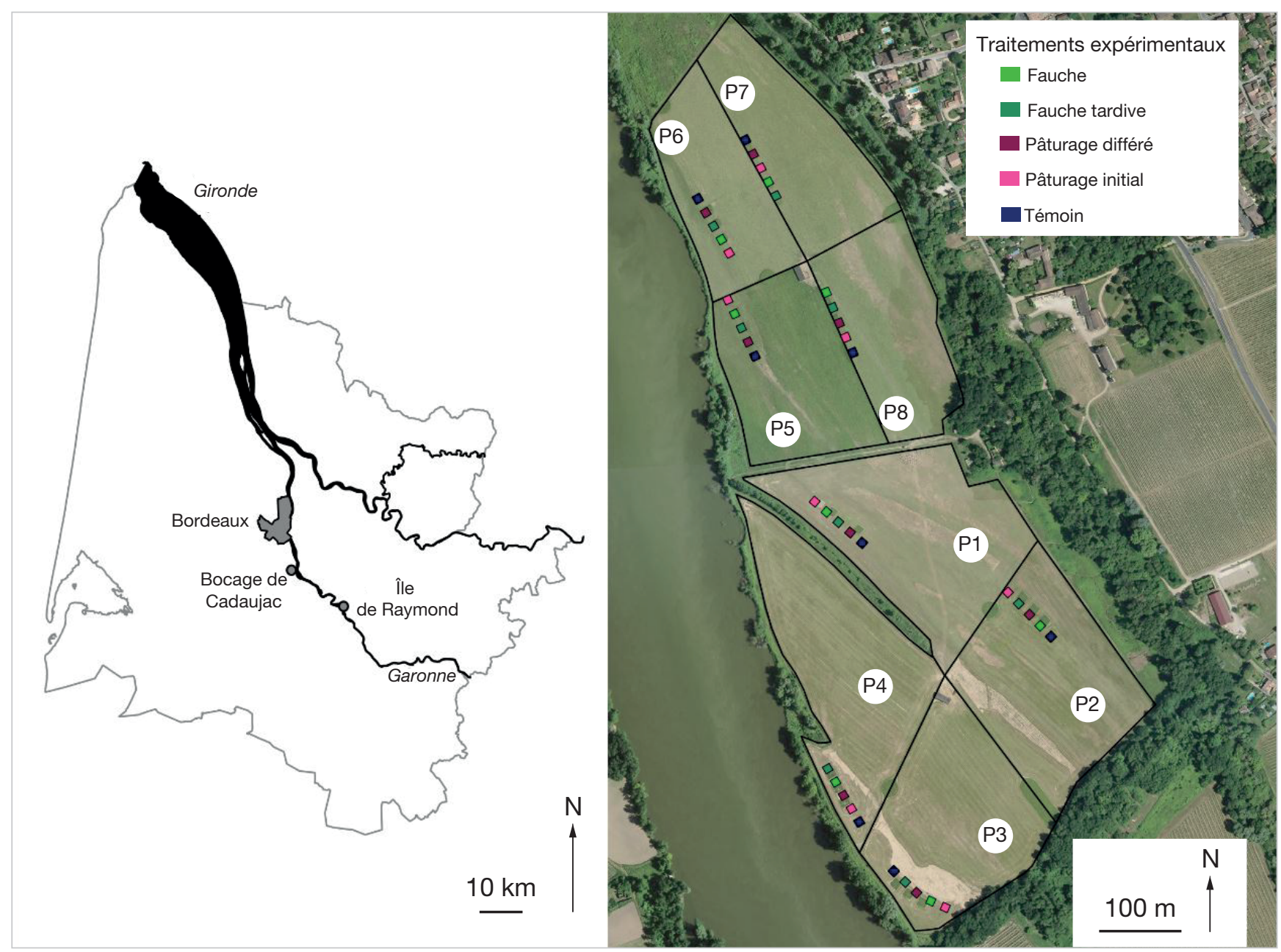

FiG. 1. - Localisation géographique de la prairie donneuse de foin (bocage de Cadaujac) et de la zone restaurée (Île de Raymond). Dispositif d'apport de foin mis en place sur l'île de Raymond, dans les huit blocs (parcs P1 à P8). Source orthophotographie: BDORTHO IGN 2015.

- l'apport de foin est essentiel dans l'initiation («origine») de la trajectoire temporelle de restauration des communautés végétales et son orientation vers la communauté de référence;

- la gestion des premiers stades de recolonisation suite à l'apport de foin est susceptible d'affecter la dynamique (direction et amplitude) de restauration via notamment la perturbation par le pâturage ou la fauche et leurs effets sur les interactions entre plantes.

Le suivi pluriannuel (sur cinq années) des communautés végétales étudiées dans cette expérimentation et leur comparaison avec la communauté de référence, une prairie alluviale gérée par fauche et pâturage et correspondant au site «donneur » dont est issu le foin transféré, doit permettre d'en décrire les trajectoires et d'attester de leur orientation ou non vers la communauté de référence. Le formalisme des résultats (choix des métriques, et descripteurs des communautés en cours de restauration et de l'état de référence) pour illustrer ce suivi, qui est un choix méthodologique fort pouvant aboutir à des résultats contradictoires (Laughlin et al. 2017), sera également exploré. Cet article s'inscrit dans le cadre des travaux présentés à l'occasion du colloque REVER 10, qui s'est tenu en mars 2019 et dont l'article introductif de Dutoit et al. (2021) rappelle les éléments saillants.

\section{MATÉRIEL ET MÉTHODES}

Le site d'expérimentation se situe sur une île fluviale régulièrement inondée en amont de Bordeaux (Île de Raymond) de 100 ha longée par le cours principal de la Garonne et séparée de la rive à l'est par un bras secondaire étroit et peu actif de la Garonne (Fig. 1). L'histoire complexe du site (Corcket et al. 2015) a abouti à une mosaïque paysagère hétérogène, au sein de laquelle une ancienne maïsiculture cultivée jusqu'en 2009 a été abandonnée puis reconvertie en prairie semée en 2010 sur 30 ha. Un mélange mixte (poacéesfabacées) de six espèces (Dactylis glomerata L., Schedonorus arundinaceus Schreb., Lolium perenne L., Lotus corniculatus L., Trifolium repens L., Trifolium hybridum L.) a été utilisé dans le cadre d'un projet de "réhabilitation" (Muller et al. 1998) avec pour objectif d'obtenir une prairie artificielle destinée à être exploitée extensivement. Cette technique a ainsi permis une restauration rapide de la fonction de production et la remise en pâturage pour une exploitation ovine (charge variable d'environ 200 brebis et leurs agneaux) dès 2011 (Corcket et al. 2015). La surface en prairie est divisée en huit parcelles (environ 4 ha chacune), généralement pâturées suc- 
cessivement en rotation de mars à octobre et préalablement fauchées pour les parcelles les plus tardivement mises en pâture. La période et la durée de passage des animaux dans chaque parcelles sont variables, dépendant notamment des conditions météorologiques et d'inondation.

Suite à cette première étape de "réhabilitation ", une expérimentation visant à tester différents types de gestion dans le cadre de la restauration de prairies alluviales a été construite sur ce site en 2014 afin de passer d'une étape de réhabilitation à une étape de restauration sensu stricto. Cinq placettes expérimentales de $100 \mathrm{~m}^{2}(10 \times 10 \mathrm{~m})$ établies dans chacune des huit parcelles à des positions topographiques similaires (soit $5 \times 8=40$ placettes expérimentales) ont été délimitées. Toutes les placettes expérimentales ont donc été semées dans le cadre de la réhabilitation de 2010 et ont fait l'objet, hormis les "témoins", d'un hersage superficiel (quelques centimètres de profondeur) avant le transfert de foin, en vue de limiter la compétition par la végétation en place et de favoriser l'installation des graines apportées par le foin. En août 2014, le transfert de foin frais a été réalisé à raison d'un ratio surfacique d'environ $3: 1$, représentant environ $1 \mathrm{~m}^{3}$ de foin apporté pour chaque placette de $100 \mathrm{~m}^{2}$. Le site donneur était une prairie alluviale riche en espèces (des relevés floristiques réalisés en 2013 ayant permis de recenser 20,3 $\pm 1,9$ espèces $m-1, n=$ 20), traditionnellement fauchée et pâturée, au sein d'un espace naturel sensible bocager localisé à Cadaujac (Gironde), à environ $15 \mathrm{~km}$ en amont du site expérimental (Fig. 1). Dans chacune des huit parcelles, une modalité de gestion testée a été assignée aléatoirement pour chacune des cinq placettes expérimentales. Les modalités sont les suivantes: témoin sans transfert de foin (T) ; et, pour les placettes ayant été soumises au transfert de foin en août 2014, pâturage initial dès le transfert de foin en 2014 (PI), pâturage différé mis en œuvre à partir de juin 2015 (PD), fauche fin juin en 2015 et les années suivantes (F), fauche tardive fin août-début septembre à partir de 2015 (FT). Les modalités T et PI n'ont pas été mises en défens, tandis que les autres modalités ont été clôturées en 2014. L'exclos du pâturage différé (PD) est ensuite supprimé fin 2015, tandis que les modalités fauchées sont restées clôturées pendant la totalité du suivi (de 2014 à 2019).

Au centre de chaque placette expérimentale $(n=40)$, les données de végétation ont été acquises sur un relevé constitué de quatre quadrats permanents de $1 \mathrm{~m}^{2}$ alignés et adjacents. Dans chaque quadrat, les relevés sont faits à l'aide de la méthode des points-contacts (Stampfli 1991) permettant de quantifier la présence des espèces végétales phanérogames au niveau de 16 points situés à l'intersection d'une grille régulière (de maille de $25 \mathrm{~cm}$ ). Le score (fréquence brute) de chaque espèce dans les $4 \mathrm{~m}^{2}$ d'un relevé est le nombre de contacts (1 à 64), toute espèce non intersectée avec un point mais à l'intérieur des $4 \mathrm{~m}^{2}$ étant notée 0,5 . Les relevés ont été réalisés tous les deux ans, en 2015, 2017 et 2019.

Afin de sélectionner la prairie donneuse, des relevés de végétation ont été réalisés dans sept prairies au sein de l'Espace Naturel Sensible (ENS) bocager de Cadaujac en 2013 (Fig. 2). À cette date, quatre relevés floristiques ont été réalisés dans la prairie donneuse. Celle-ci a fait l'objet d'une seconde cam- pagne de relevés floristiques en 2017 (huit relevés floristiques réalisés à cette date) en vue de caractériser la dynamique temporelle de la communauté végétale de référence. L'objectif principal de ces relevés étant l'identification des espèces végétales constitutives de la communauté de référence et non un suivi fin de la dynamique temporelle de restauration de la végétation, les relevés de végétation réalisés dans la prairie donneuse étaient constitués de cinq quadrats de $1 \mathrm{~m}^{2}$ répartis sur une surface de $4 \times 4 \mathrm{~m}$ qui constitue chaque relevé, dans lesquels l'abondance de chaque espèce est quantifiée sur la base des coefficients d'échelle de dominance (Hill et al. 2005; Alfonsi et al. 2017).

\section{TRAITEMENT ET ANALYSE DES DONNÉES}

Un premier traitement de la base de données floristiques a été réalisé afin d'homogénéiser la liste des taxons recensés dans les différents relevés floristiques (mise à jour des noms d'espèces et suppression des synonymies, regroupement de taxons pouvant avoir fait l'objet de confusions, ou dans le cas de taxons d'un même genre identifiés à des niveaux taxonomiques différents - spécifique ou générique; Annexe 1).

Les richesses spécifiques totales de chaque relevé floristique (RS; le nombre d'espèces recensées) ont été calculées. L'effet de la modalité de gestion et de l'année sur la richesse spécifique a été testé à l'aide d'un modèle linéaire généralisé (résidus suivant une loi de Poisson) mixte (GLMM) avec la date, la gestion et leur interaction comme effets fixes et la parcelle comme facteur aléatoire. Le modèle le plus parcimonieux (sur la base de l'AICc) a été conservé et un test de contrastes a été réalisé pour les comparaisons multiples.

L'abondance relative Aik de chaque espèce i dans chaque relevé $\mathrm{k}$ a été calculée comme suit:

Aik $=$ Nik / $\sum \mathrm{i}=1 \mathrm{~S}$ Nik (éq. 1$)$

où Nik est l'abondance (fréquence brute ou coefficient d'échelle de dominance) de l'espèce i dans le relevé $\mathrm{k}$ et $\mathrm{S}$ est la richesse spécifique dans le relevé $\mathrm{k}$.

La comparaison des compositions floristiques entre communautés végétales restaurées et de référence, et la représentation des trajectoires des communautés restaurées a été réalisée selon deux méthodes. Premièrement une ordination directe par projection des abondances relatives Aik moyennes, par traitement et par année, dans un diagramme original, que nous avons nommé «TERNAIRE» («Triangle d'Évaluation de la ReNAturation et des Itinéraires de Restauration Écologique») construit sur la base des contributions de trois groupes d'espèces:

- les espèces semées en 2010 caractérisant la situation initiale théorique de la restauration suite à la réhabilitation (Isp);

- les espèces identifiées dans les relevés du site de référence (relevés de 2013 et de 2017), caractérisant l'apport théorique par transfert de foin (Rsp);

- les autres espèces n'appartenant à aucun des deux groupes précédents et caractérisant le pool d'espèces latentes et celles du paysage $(\mathrm{Osp})$, liées respectivement à la banque et la pluie de graines (Alard et al. 1998). 


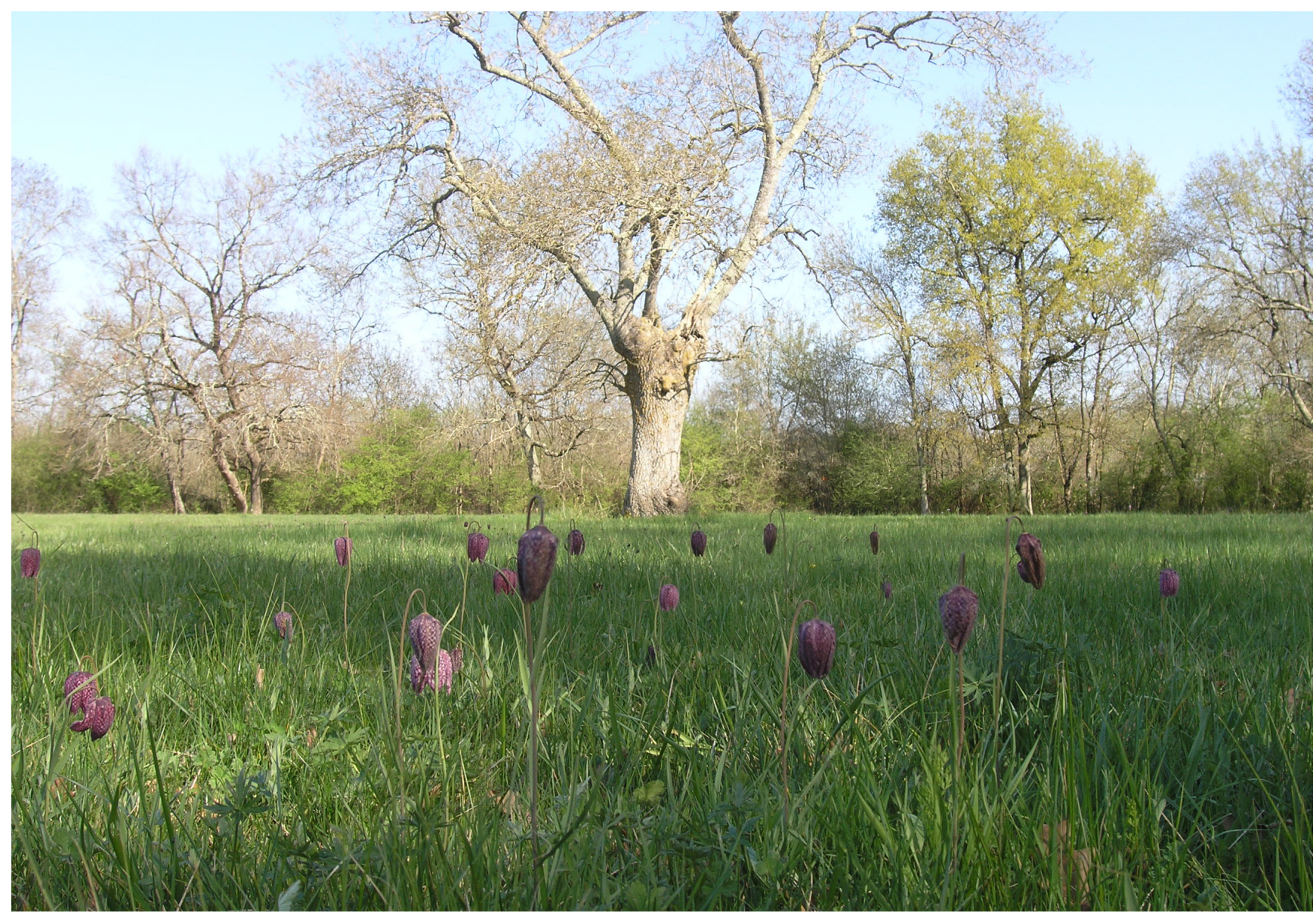

FiG. 2. - Prairie alluviale de référence, donneuse de foin située à Cadaujac (Gironde). Crédit photo: Didier Alard.

Deuxièmement, des ordinations indirectes par analyse multivariée, de type NMDS (Non Metric Multidimensional Scaling) ont été appliquée à la matrice [relevés $\times$ espèces] des abondances relatives Aik.

Ce type d'analyse permet une ordination dans laquelle la distance entre les relevés est calculée itérativement pour converger avec l'ordre de rang des dissimilarités floristiques entre ces relevés (Austin 2005). Nous avons utilisé la distance de Bray-Curtis pour calculer cette dissimilarité, la projection des relevés le long des axes de la NMDS reflétant cette distance. Deux analyses successives ont été réalisées après avoir supprimé de la base de données les observations non déterminées au niveau spécifique ou générique, ainsi que les taxons recensés une seule fois (Annexe 1): une première sur un jeu de données comprenant uniquement les placettes expérimentales pour les années 2015,2017 et $2019(3 \times 40$ placettes $\times 1$ année) afin de mesurer les variations de compositions floristiques intra-traitements (hétérogénéité annuelle entre placettes d'un même traitement), inter-traitements (effets des modalités expérimentales) et interannuelles (dynamiques des compositions). Une seconde analyse NMDS a été réalisée sur l'ensemble du jeu des données expérimentales (40 placettes $\times 3$ années) en intégrant également les relevés floristiques du site de référence (années 2013 et 2017).
Toutes les analyses ont été réalisées sous $\mathrm{R}$ (version 3.6.3., R Core Team 2020). Le GLMM a été réalisé avec la fonction glmer du paquet lme4 (Bates et al. 2015), la sélection du modèle le plus parcimonieux avec la fonction dredge du paquet $\mathrm{MuMin}$ (Bartoń 2020) et les tests de contrastes avec la fonction emmeans du paquet emmeans (Lenth 2020). Les NMDS ont été réalisées à l'aide du paquet vegan (Oksanen et al. 2010). Les figures ont été élaborées à l'aide des paquets ade4 (Chessel et al. 2004), ggplot2 (Wickham 2016) et ggtern (Hamilton \& Ferry 2018).

\section{RÉSULTATS}

Après traitement de la base de données, 108 taxons ont été détectés (Annexe 1), dont 96 sur l'ensemble des placettes expérimentales et 46 dans la prairie donneuse. Le modèle le plus parcimonieux expliquant les variations de richesses spécifiques inclut la date et les modalités de gestion, mais pas l'interaction des deux facteurs. Les richesses moyennes des placettes (Fig. 3) varient de $8,6 \pm 1,1$ à 21,4 \pm 7,6 espèces en 2015, en fonction des traitements expérimentaux, et augmentent significativement entre 2015 et 2019 avec des valeurs en 2019 variant de $14,7 \pm 3,5$ à $23,7 \pm 2,8$ espèces. Les rangs des traitements restent exactement les mêmes 


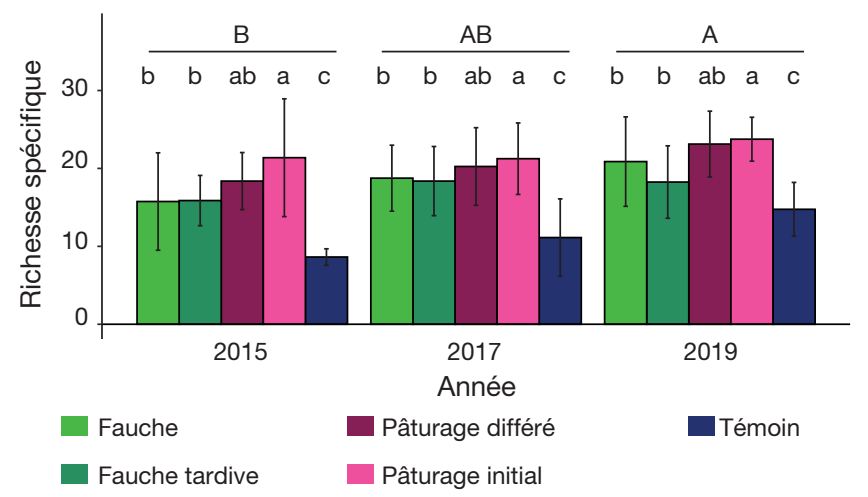

FIG. 3. - Niveaux de richesse spécifique en plantes vasculaires (avec écarttype) dans le dispositif expérimental d'apport de foin, en 2015, 2017 et 2019 ( $\mathrm{n}=8$ pour chaque modalité). Les lettres majuscules indiquent les différences significatives entre années, les lettres minuscules indiquent les différences significatives entre traitements expérimentaux.

durant l'expérimentation, les témoins étant systématiquement les moins riches, quelle que soit l'année. La modalité de pâturage initiale est toujours significativement plus riche que les modalités fauchées.

Après simplification du jeu de données (suppression des taxons non déterminés ou observés une seule fois), la première NMDS (données 2015-2019 du site expérimental) a été conduite sur la matrice des 120 relevés floristiques (40 placettes expérimentales $\times 3$ dates) et 70 espèces (Annexe 1). Le gradient floristique représenté par l'axe NMDS1 traduit le statut des espèces: les six espèces semées lors de la phase initiale de réhabilitation (Isp) sont projetées dans la partie négative de l'axe, tandis que 23 des 28 espèces de référence présentes dans ce jeu de données (soit $82 \%$ ) sont projetées dans la partie positive de l'axe, les autres espèces (Osp) étant réparties sur l'ensemble de l'axe (Annexe 2). La répartition des espèces, en fonction de leur groupe, le long de l'axe NMDS2 est moins marquée mais on peut noter que la partie positive de l'axe correspond essentiellement à des espèces "autres" (Osp) et la partie négative à des espèces «autres » et de référence (Annexe 2). L'axe NMDS1 exprime l'effet traitement (témoin $v$ s tous les autres traitements) et l'effet annuel, avec un glissement temporel vers la partie positive de l'axe, et ce, malgré une hétérogénéité intra-traitement et intra-annuelle relativement importante le long de cet axe (Fig. 4). L'axe NMDS2 traduit essentiellement l'hétérogénéité propre à chaque modalité (i.e. intra-traitement et intra-annuelle). Cette hétérogénéité est variable: elle est minimale pour les placettes témoin en 2015 et 2017 et, pour les autres traitements, variable en fonction de l'année. On note cependant une augmentation de cette hétérogénéité intra-traitement en 2019 (Fig. 4).

L'ordination directe dans un référentiel construit a priori sur les trois groupes d'espèces (référence/semis/autres; représentation TERNAIRE) permet de déterminer les trajectoires des cinq traitements en terme d'abondances relatives de ces groupes d'espèces dans les relevés (Fig. 5A). En 2015 et 2017, les placettes témoins sont composées des six espèces utilisées pour la phase semis (Isp) qui représentent une abondance relative de $78 \pm 11$ (écart-type) $\%$ en 2015 et $75 \pm 14 \%$ en 2017 avec en plus une proportion d'environ $19 \%$ d'espèces de référence (Rsp) et autour de $5 \%$ d'autres espèces (Osp). Sur les modalités dans lesquelles le transfert de foin a été opéré, l'abondance relative des espèces du groupe de référence varie entre $26 \pm 10 \%$ (traitement FT) et $45 \pm 23 \%$ (traitement PI) en 2015. Pour l'ensemble des modalités, sauf le témoin, et de façon moindre pour le traitement "fauche», l'orientation et l'amplitude de la trajectoire entre 2015 et 2017 traduit un renforcement dans la composition du groupe d'espèces du site de référence, ceci au détriment des espèces initialement présentes dans le semis (Isp). Ces dernières ne représentent plus que la moitié (en 2015) voire le tiers (en 2017) du cortège exprimé dans les placettes. Par la suite, les trajectoires de toutes les modalités entre 2017 et 2019, y compris les témoins, montrent une inflexion dans l'amplitude et surtout la direction au bénéfice des «autres » espèces qui en 2019 représentent en moyenne, tous traitements confondus, $23 \pm 13 \%$ des espèces exprimées.

La seconde NMDS, réalisée pour la période 2015-2019, sur les données du site expérimental et du site de référence (pour ce dernier, années 2013 et 2017), montre une discrimination claire des placettes expérimentales et des relevés de la prairie source le long de l'axe NMDS1 (Fig. 5B). En effet, le gradient floristique représenté par cet axe traduit bien la discrimination entre les espèces issues du semis initial (Isp, toutes projetées dans la partie positive de l'axe) et les espèces de référence (Rsp, dont plus de $80 \%$ sont projetées sur la partie négative de l'axe, Annexe 3). Les autres espèces (Osp) sont quant à elles principalement projetées sur la partie positive de l'axe (Annexe 3). Le gradient floristique représenté par l'axe NMDS2 ne semble pas lié à l'appartenance des espèces à l'un de ces trois groupes (Annexe 3). Le plan factoriel NMDS1 - NMDS2 montre, en ce qui concerne les trajectoires des placettes expérimentales, l'effet du transfert de foin sur le turn-over des espèces en direction de la composition spécifique du site de référence (position des traitements inoculés décalés vers la partie négative de l'axe NMDS1, en comparaison avec le témoin), la dynamique temporelle de tous les traitements vers le site de référence (i.e. le long de l'axe NMDS1), avec toutefois une plus grande amplitude dans la dynamique des placettes pâturées le long de cet axe NMDS1, alors que la dynamique temporelle des placettes fauchées s'exprime de manière tout aussi marquée le long de l'axe NMDS2, et la dynamique temporelle propre du site donneur de référence, qui s'exprime dans une direction opposée à celle du site expérimental (vers la partie négative de l'axe NMDS2). Une étude de la composition spécifique des relevés du site donneur de référence montre, entre 2013 et 2017, une augmentation de l'abondance relative de certaines espèces (Agrostis L. sp., Ranunculus L. sp., Schedonorus arundinaceus), voire une détection de nouvelles espèces (e.g., Convolvulus arvensis L., Cynodon dactylon L., Linum usitatissimum subsp. angustifolium L., Lotus corniculatus, Poa pratensis L., Stellaria graminea L. ou encore Taraxacum officinale H.Wiggg. s.l.) au détriment de l'abondance relative d'espèces prairiales caracté- 


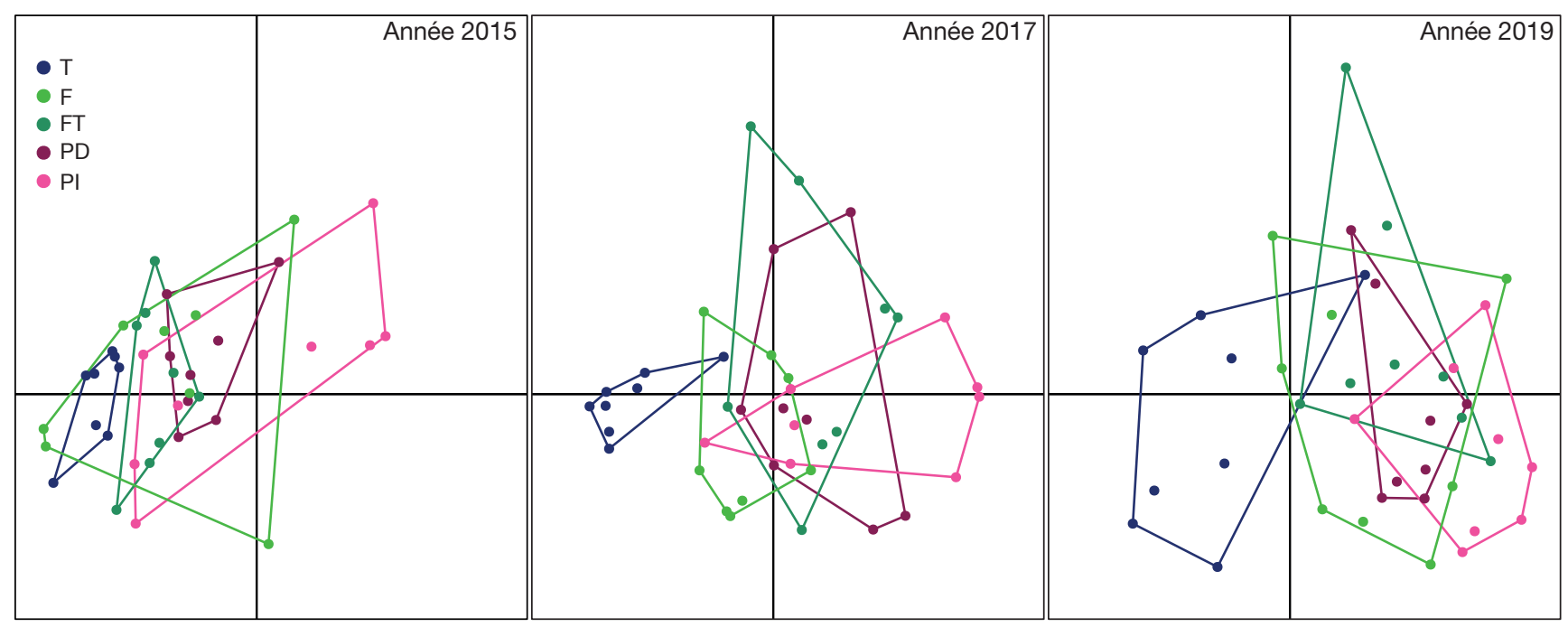

FIG. 4. - Plans factoriels des analyses NMDS (1,2) sur la composition floristique 2015, 2017 et 2019 des 40 placettes suivies sur le dispositif expérimental après apport de foin. Abréviations: F, fauche; FT, fauche tardive; PD, pâturage différé; PI, pâturage initial; T, témoin.

ristiques telles que les Poaceae Bromus L. sp., Cynosurus cristatus L., Gaudinia fragilis Beauv., Geranium dissectum L., Holcus lanatus L., Hordeum secalinum Schreb., Lolium perenne, Poa trivialis L., les Fabaceae Lotus glaber Mill., Lotus pedunculatus Cav., Trifolium patens Schreb., Trifolium pratense L., ou encore des espèces plus rares telles qu'Anacampsis laxifolia Lam. (non détectée en 2017).

\section{DISCUSSION}

\section{EFFET DU TRANSFERT DE FOIN}

\section{ET DES TECHNIQUES ASSOCIÉES}

Quatre années après la réhabilitation initiale, les espèces semées en 2010 (e.g., Schedonorus arundinaceus, Dactylis glomerata) restent encore majoritaires dans les placettes en début d'expérimentation, même si leur cortège s'est enrichi d'un certain nombre d'espèces issues de la banque ou de la pluie de graines (e.g. Poa trivialis, Sonchus asper L.). Ce mécanisme d'enrichissement spontané après le dernier labour, tel que nous l'observons dans les placettes témoins, est bien connu dans les prairies temporaires et a été décrit comme un gain de diversité, via la banque de graines du sol puis la pluie de graines, au profit d'espèces typiquement prairiales ou d'adventices (Austrheim \& Olsson 1999). L'opération de transfert de foin, assimilable à une pluie de graines artificielle (Hutchings \& Booth 1996a) et qui avait pour but de contrebalancer l'isolement du site expérimental vis-à-vis des sources potentielles d'espèces, a initié un changement net dans la végétation. Les placettes ayant reçu le foin, en comparaison des placettes témoin, montrent des cortèges plus riches, mais ceci essentiellement du fait de la plus forte contribution des espèces «autres», caractérisant les habitats de friches post-culturales. Paradoxalement, le premier effet du transfert de foin semble donc être l'intensification de l'enrichissement spontané décrit précédemment, à partir essentiellement de la banque de graines du sol et au bénéfice d'espèces adventices dont les graines sont particulièrement longévives (Dutoit \& Alard 1995). L'explication est sans doute à rechercher dans l'application du hersage du sol, qui a été pratiqué sur toutes les placettes recevant du foin du site donneur, à l'exception des témoins. Ces résultats suggèrent que le hersage superficiel a généré, comme attendu, des conditions favorables pour le recrutement des graines présentes (niche de régénération sensu, Grubb 1977), en ouvrant la végétation en place et en réduisant ainsi ses capacités de compétition, qui ont bénéficié à la fois aux graines transférées via le foin mais surtout, dans un premier temps, à la banque de graines héritée de la période cultivée avant 2010 (Hofmann \& Isselstein 2004; Török et al. 2011). La technique de transfert de foin est considérée comme particulièrement efficace dans des milieux comme les prairies de fauche (Sullivan et al. 2020) et les pelouses calcicoles (Kiehl \& Pfadenhauer 2007), où elle permet d'approcher les $60 \%$ de similarité entre les sites donneurs et receveurs. Une étude comparative sur la restauration des zones humides (Klimkowska et al. 2007) montre, quand le transfert de foin est associée à l'étrépage superficiel du sol, que le succès de restauration (gain de similarité vis-à-vis du pool d'espèces de la communauté cible) est encore amélioré. Le hersage, dans notre expérimentation, a joué ce rôle de création de niche de régénération en ouvrant une "fenêtre d'opportunité" pour la colonisation, au bénéfice d'espèces adventices déjà en place dans la banque de graines et qui représentent plus du tiers des espèces exprimées en 2015.

\section{EFFET DES GESTIONS DIFFÉRENCIÉES}

L'effet spécifique lié au transfert de foin est, quant à lui, révélé par la gestion différenciée des placettes au cours de la première année d'expérimentation. Le pâturage est susceptible d'affecter le succès de la régénération et la survie des plantules via la défoliation et le piétinement qui lui sont associés (Belsky 1992; Lagendijk et al. 2017) et la constitution des 
A

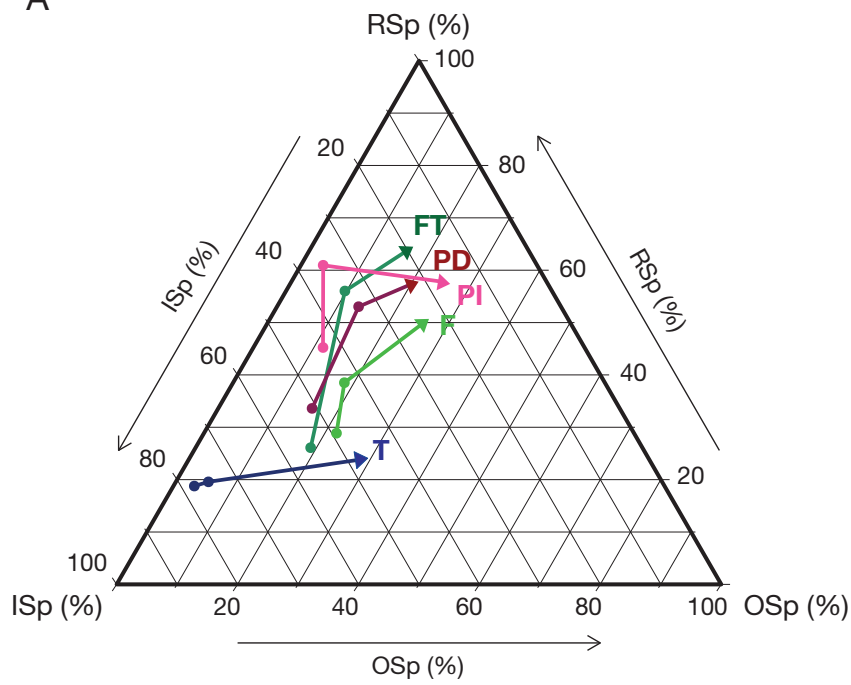

B

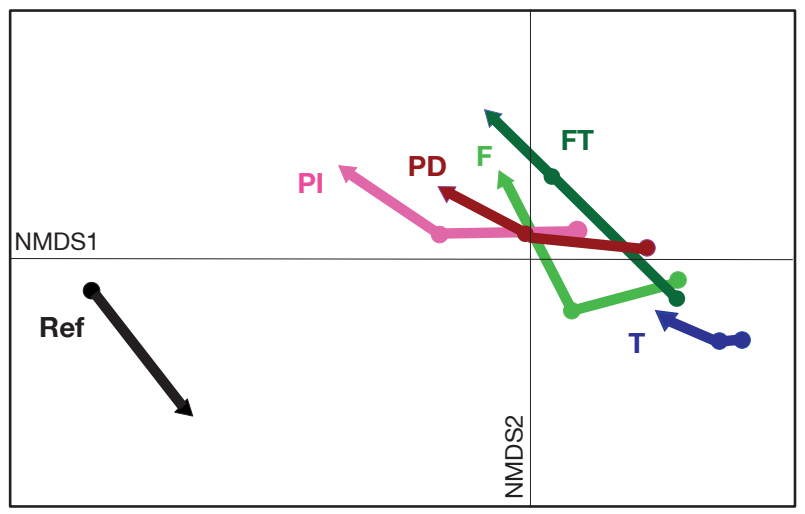

FIG. 5. - Trajectoires temporelles des communautés restaurées en fonction des modalités. A, représentation «TERNAIRE» pour les trajectoires temporelles 2015-2017-2019; B, représentation en plan factoriel NMDS pour les trajectoires temporelles 2015-2017-2019 sauf pour le site de référence (2013-2017). RSp, ISp et OSp représentent respectivement les trois groupes d'espèces de la prairie de référence, du semis initial et autres. Abréviations: $\mathbf{F}$, fauche; FT, fauche tardive; PD, pâturage différé; PI, pâturage initial; T, témoin.

exclos visait à garantir le succès du transfert de foin dans les premiers stades, a minima dans les premier mois après transfert pour le pâturage différé, davantage pour les modalités associées à la fauche. Paradoxalement, c'est la dernière combinaison, c'est à dire le pâturage initial sans exclos, qui constitue la seule modalité montrant, dès 2015, la plus forte proportion en espèces du groupe de référence, apportées par le foin. Les effets de ce pâturage, quelques mois après le transfert (août à octobre 2014), peuvent s'expliquer par deux processus : d'une part les graines transférées par le foin n'ont pas encore germé à cette période et la défoliation n'a pas affecté les espèces à ce stade de développement ; d'autre part le piétinement a probablement favorisé la germination des graines en les intégrant avec la litière au sol (Winkel \& Roundy 1991), le pâturage permettant par ailleurs le contrôle de la végétation en place lors de la germination (Hutchings \& Booth 1996b). Le maintien d'une fenêtre d'opportunité pour les espèces transférées est davantage assuré par le pâturage initial, qui a maximisé les chances d'établissement de la végétation transférée, plutôt que par toutes les autres modalités de gestion, pour lesquelles l'exclusion de la perturbation principale n'a pas eu l'effet escompté (Balke et al. 2014).

La gestion sur le plus long terme (cinq années) est un déterminant primordial de la direction et de l'amplitude des trajectoires de végétation à partir du transfert de foin. Alors qu'en 2017 les placettes témoins sont encore remarquablement stables et peu diversifiées, la forte dynamique des autres placettes varie selon le type de gestion (fauche, pâture) et son calendrier. Dans les placettes pâturées, l'effet des micro-perturbations par les animaux qui maintiennent les espèces compétitrices et ré-initient les niches de régénération (Grubb 1977) expliquent le gain en espèces cibles de la communauté de référence, qui deviennent dominantes dans le cortège floristique. La modalité de fauche tardive, après la saison de végétation, montre une trajectoire assez similaire à celles des modalités de pâturage à l'inverse de la modalité de fauche classique, plus précoce, dont la trajectoire est beaucoup moins prononcée en début d'expérimentation. La fauche diminue la biomasse sur pied et la compétition de la végétation en place sur les plantules et elle est donc susceptible d'améliorer la régénération des espèces (John et al. 2016). Mais le calendrier de fauche est également primordial pour expliquer ces effets, d'une part sur les phénophases fleuries (Johansen et al. 2019) et en conséquence la production de graines, d'autre part sur la levée de compétition, plus importante en fauche précoce (i.e. pendant la période de végétation). Le différentiel entre nos deux modalités de fauche suggère que la fauche tardive, en permettant une pluie de graines par la végétation installée, maintient un flux d'entrées de propagules au bénéfice des espèces initialement transférées par le foin, ce que ne permet pas une fauche trop précoce qui s'effectue avant que de nombreuses espèces aient fleuri. L'apport de graines sous toutes ses formes apparaît ainsi comme le processus clé de la restauration, dominant les effets éventuels des interactions biotiques ou des perturbations. L'augmentation générale de l'hétérogénéité intra-modalités entre 2015 et 2017 souligne, quant à elle, le poids des contingences locales et de la stochasticité des processus démographiques (Silvertown 1981). La dynamique à partir de 2017 montre une inflexion générale pour toutes les modalités, y compris cette fois pour le témoin, au bénéfice des espèces "autres" et aboutissant à une déviation de la trajectoire vers le système de référence. Le transfert de foin étant un événement ponctuel, le pool d'espèces apportées s'exprime lors des premières années mais doit être renouvelé au cours des années. Or ce pool d'espèces contenu dans le foin transféré n'est pas une représentation complète de la communauté d'origine. Les nouvelles espèces intégrant la communauté viennent donc probablement de la 
pluie de graines, différente de la communauté de référence, étant donné l'isolement du site restauré. Cette dynamique à plus long terme suggère cette fois-ci le rôle de la stochasticité environnementale inter-annuelle d'origine climatique ou liée à la gestion du site à laquelle sont associés les stocks de la banque de graines du sol ou de la pluie de graines locale.

\section{CONCLUSION}

Nous avons choisi de représenter les trajectoires de restauration via deux formalismes: la représentation nouvelle TERNAIRE, séparant les cortèges en trois groupes a priori (les espèces réintroduites par la réhabilitation, la communauté de référence, les espèces spontanées "autres») et privilégiant le critère de présence des espèces et l'analyse multivariée basée sur les dissimilarités (NMDS) construisant des gradients floristiques a posteriori et basée sur les abondances des espèces. Les résultats communs aux deux représentations soulignent la faiblesse de la restauration passive (colonisation spontanée des placettes témoin), le rôle de la banque de graines du sol dans cette recolonisation (révélée par le hersage superficiel et sans doute l'effet inter-annuel), l'efficacité du transfert de foin pour se rapprocher de la communauté de référence, ainsi que les effets différentiels de la gestion, désignant au passage le pâturage initial comme une voie particulièrement efficace dans la restauration. En revanche, les trajectoires des placettes, dans leurs directions et leurs amplitudes, apparaissent fortement liées au formalisme mettant en jeu des métriques (présence vs abondance) et des espaces de référence (a priori vs a posteriori) différents. Par exemple l'accent mis sur la présence des espèces souligne la similitude des trajectoires et le meilleur résultat de restauration des modalités PI et FT, tandis que l'abondance au sein des cortèges sépare les trajectoires des modalités pâturées vis-à-vis de celles des modalités fauchées. Une différence cruciale dans les deux formalismes provient directement de la construction de l'espace de référence, qui est fixé dans le modèle triangulaire mais dépendant du jeu de données dans l'analyse multivariée. Une conséquence forte est que la communauté de référence possède sa propre dynamique dans le second formalisme (NMDS) alors qu'elle est fixée quant à son cortège dans le modèle TERNAIRE. Si les deux modèles permettent de mesurer "l'écart à la référence", considéré comme une évaluation du succès de restauration (Jaunatre et al. 2013), l'analyse plus fine des trajectoires devrait bénéficier du développement de nouveaux outils d'analyse de la dynamique des communautés (De Càceres et al. 2019).

\section{Remerciements}

Cette étude a été financée par Bordeaux Métropole, le département de la Gironde et l'Agence de l'Eau Adour Garonne. Elle a également bénéficié des supports du Ministère de l'Ecologie, du Développement durable, des Transports et du Logement (AAP «Restauration de milieux remarquables ou sensibles " de la SNB), du Ministère de l'Enseignement et de la Recherche (bourse doctorale de MG) et de la Communauté de Communes «Convergence Garonne». Nous remercions pour leur assistance scientifique et/ou technique: A. Bischoff,
P. Massart, M. Thierry, A. Castagnos, I. van Halder, J. Chenot, A. et M. Corcket et l'AMAP de l'Artolie. Merci aux relectrices de leurs suggestions et conseils judicieux pour améliorer une première version.

\section{RÉFÉRENCES}

Alard D., Poudevigne I., Dutoit T. \& Decaens T. 1998. Dynamics of biodiversity in a changing space - The case of chalk grasslands in the lower section of the Seine Valley. Acta Oecologica 19: 275-284.

Alard D., Bourcier A., Bureau F., Lefebvre D., Mesnage V. \& POUDEVIGNE I. 2002. - Zones humides de la basse vallée de la Seine. Programme scientifique Seine-Aval. Quae, Versailles, 36 p.

Alfonsi E., Benot M.-L., Fievet V. \& Alard D. 2017. — Addressing species turnover and community changes in vegetation resurvey studies. Applied Vegetation Science 20: 172-182. https://doi. org/10.1111/avsc. 12258

AUSTIN M. P. 2005. - Vegetation and environment: discontinuities and continuities, in VAN Der MAarel E. (éd.), Vegetation Ecology. Blackwell Publishing, Oxford: 52-84.

Austrheim G. \& Olsson E. G. A. 1999. - How does continuity in grassland management after ploughing affect plant community patterns? Plant Ecology 145: 59-74. https://doi. org/10.1023/A:1009804509239

Balke T., Herman P. M. J. \& Bouma T. J. 2014 - Critical transitions in disturbance-driven ecosystems: identifying windows of opportunity for recovery. Journal of Ecology 102: 700-708. https://doi.org/10.1111/1365-2745.12241

BARNAUD G. 1998. - Conservation des zones humides; concepts et méthodes appliquées à leur caractérisation. MNHN, Paris, $451 \mathrm{p}$.

BARTOŃ K. 2020. - MuMIn: Multi-Model Inference. R package version 1.43.17. https://CRAN.R-project.org/package=MuMIn, dernière consultation le 10 juillet 2020.

Bates D., Maechler M., Bolker B. \& Walker S. 2015. - Fitting linear mixed-effects models using lme4. Journal of Statistical Software 67: 1-48. https://doi.org/10.18637/jss.v067.i01

BAYLEY P. B. 1995. - Understanding large river-floodplain ecosystems: significant economic advantages and increased biodiversity and stability would result from restoration of impaired systems. BioScience 45: 153-158. https://doi.org/10.2307/1312554

BELSKY A. J. 1992 - Effects of grazing, competition, disturbance and fire on species composition and diversity in grassland communities. Journal of Vegetation Science 3: 187-200. https://doi. org/10.2307/3235679

Chessel D., Dufour A. \& Thioulouse J. 2004. — The ade 4 Package - I: One-Table Methods. R News 4 (1): 5-10.

Corcket E., Benot M. L., Bischoff A., Poncin G. \& Henriot L. 2015. - Restauration écologique d'une ancienne maïsiculture en zone alluviale: l'Île de Raymond en bord de Garonne. Penn ar Bed 220: 3-9.

De Cáceres M., Coll L., Legendre P., Allen R. B., Wiser S. K., Fortin M.-J., Condit R. \& Hubbell S. 2019. - Trajectory analysis in community ecology. Ecological Monographs 89: e01350. https://doi.org/10.1002/ecm.1350

DUfoUR S. \& PIÉGAY H. 2009. — From the myth of a lost paradise to targeted river restoration: forget natural references and focus on human benefits. River Research and Applications 25: 568-581. https://doi.org/10.1002/rra.1239

DuTOIT T. \& ALARD D. 1995 - Permanent seed banks in chalk grassland under various management regimes: their role in the restoration of species-rich plant communities. Biodiversity and Conservation 4:939-950. https://doi.org/10.1007/bf00058205

Dutoit T., Gallet S. Heckenroth A. \& Buisson É. 2021. 2008-2019, plus d'une décennie d'échanges et de débats autour de la restauration écologique en France, in Gourdain P. (éd.), 
REVER 10 - 10ème Colloque du Réseau d'Échange et de Valorisation en Écologie de la Restauration, Paris, 19-21 mars 2019. Naturae 2021 (19): 271-276. https://doi.org/10.5852/ naturae2021a19

Engst K., BaAsch A., Erfmeier A., Jandt U., May K., Schmiede R. \& BRUELHEIDE H. 2016. - Functional community ecology meets restoration ecology: assessing the restoration success of alluvial floodplain meadows with functional traits. Journal of Applied Ecology 53: 751-764. https://doi.org/10.1111/1365-2664.12623

FATTORINi M. \& HALLE S. 2004. - The dynamic environmental filter model: how do filtering effects change in assembling communities after disturbance?, in TEMPERTON V. M., HOBBS R. J., Nuttle T. \& Halle S. (éds), Assembly Rules and Restoration Ecology. Island Press, Washington: 96-114.

GRUBB P. J. 1977. - The maintenance of species richness in plant communities: the importance of the regeneration niche. Biological Review 52: 107-145. https://doi.org/10.1111/j.1469185X.1977.tb01347.x

HAMILTON N. E. \& FerRY M. 2018. — ggtern: ternary diagrams using ggplot2. Journal of Statistical Software 87: 1-17. https:// doi.org/10.18637/jss.v087.c03

Henle K., Alard D., Clitherow J., Cobb P., Firbank L., Kull T., Mccracken D., Moritz R. F. A., Niemela J., Rebane M., Wascher D., Watt A. \& Young J. 2008. — Identifying and managing the conflicts between agriculture and biodiversity conservation in Europe - A review. Agriculture Ecosystems \& Environment 124: 60-71. https://doi.org/10.1016/j.agee.2007.09.005

Hill D., Fasham M., Tucker G., Shewry M. \& Shaw P. (éds) 2005. - Handbook of Biodiversity, Methods: Survey, Evaluation and Monitoring. Cambridge University Press, 573 p.

HOFMANN M. \& ISSELSTEIN J. 2004. - Seedling recruitment on agriculturally improved mesic grassland: the influence of disturbance and management schemes. Applied Vegetation Science 7: 193-200. https://doi.org/10.1111/j.1654-109X.2004.tb00610.x

HuTCHINGS M. J. \& BOOTH K. D. 1996a. - Studies on the feasibility of re-creating chalk grassland vegetation on ex-arable land. I. The potential roles of the seed bank and the seed rain. Journal of Applied Ecology 33: 1171-1181. https://doi.org/10.2307/2404696

HutChings M. J. \& Booth K. D. 1996b. - Studies of the feasibility of re-creating chalk grassland vegetation on ex-arable land. II. Germination and early survivorship of seedlings under different management regimes. Journal of Applied Ecology 33: 1182-1190. https://doi.org/10.2307/2404697

Jaunatre R., Buisson E., Muller I., Morlon H., Mesléard F. \& DutorT T. 2013 - New synthetic indicators to assess community resilience and restoration success. Ecological Indicators 29: 468-477. https://doi.org/10.1016/j.ecolind.2013.01.023

Johansen L., Westin A., WeHn S., Iuga A., Ivascu C. M., KalLIONIEMI E. \& LENNARTSSON T. 2019. - Traditional seminatural grassland management with heterogeneous mowing times enhances flower resources for pollinators in agricultural landscapes. Global Ecology and Conservation 18: e00619. https:// doi.org/10.1016/j.gecco.2019.e00619

John H., Dullau S., Baasch A. \& Tischew S. 2016. - Reintroduction of target species into degraded lowland hay meadows: how to manage the crucial first year? Ecological Engineering 86: 223-230. https://doi.org/10.1016/j.ecoleng.2015.11.001

KieHL K. \& PfadenHauer J. 2007. — Establishment and persistence of target species in newly created calcareous grasslands on former arable fields. Plant Ecology 189: 31-48. https://doi.org/10.1007/ s11258-006-9164-x

Klimkowska A., Van Diggelen R., Bakker J. P. \& GrootJANS A. P. 2007. - Wet meadow restoration in Western Europe: a quantitative assessment of the effectiveness of several techniques. Biological Conservation 140: 318-328. https://doi.org/10.1016/J. BIOCON.2007.08.024

Lagendijk D. D. G., Howison R. A., Esselink P., Ubels R. \& SMIT C. 2017. - Rotation grazing as a conservation manage- ment tool: vegetation changes after six years of application in a salt marsh ecosystem. Agriculture, Ecosystems \& Environment 246 361-366. https://doi.org/10.1016/j.agee.2017.05.023

Laughlin D. C., Strahan R. T., Moore M. M., Fulé P. Z., Huffman D. W. \& Covington W. W. 2017. — The hierarchy of predictability in ecological restoration: are vegetation structure and functional diversity more predictable than community composition? Journal of Applied Ecology 54: 1058-1069. https:// doi.org/10.1111/1365-2664.12935

LENTH R. 2020. — emmeans: Estimated Marginal Means, aka LeastSquares Means. R package version 1.4.8. https://CRAN.R-project. org/package $=$ emmeans, dernière consultation le 8 février 2020 .

MChergui C., Aubert M., Buatois B., AKPa-Vinceslas M., LaNglois E., Bertolone C., Lafite R., Samson S. \& Bureau F. 2014. - Use of dredged sediments for soil creation in the Seine estuary (France): importance of a soil functioning survey to assess the success of wetland restoration in floodplains. Ecological Engineering 71: 628-638. https://doi.org/10.1016/j. ecoleng.2014.07.064

Moreno-Mateos D., Power M. E., Comín F. A. \& YockTeng R. 2012. - Structural and functional loss in restored wetland ecosystems. PLOS Biology 10: e1001247. https://doi.org/10.1371/ journal.pbio. 1001247

Muller S., Dutoit T., Alard D. \& Grevilliot F. 1998. — Restoration and rehabilitation of species-rich grassland ecosystems in France: a review. Restoration Ecology 6: 94-101. https://doi. org/10.1046/j.1526-100x.1998.06112.x

OKSANEN J., Blanchet F. G., Kindt R., LEgENdRe P., O'HARA R. Simpson G. L., Solymos P., Stevens M. H. H. \& Wagner H. 2010. - Vegan: Community Ecology Package. R package version 1.17-4. https://cran.r-project.org/package=vegan, dernière consultation le 8 février 2020.

Paillex A., Dolédec S., Castella E. \& Mérigou S. 2009. — Large river floodplain restoration: predicting species richness and trait responses to the restoration of hydrological connectivity. Journal of Applied Ecology 46: 250-258. https://doi.org/10.1111/j.13652664.2008.01593.x

PoudeVigne I., Alard D., Leuven R. \& Nienhuis P. H. 2002. A systems approach to river restoration: a case study in the Lower Seine Valley, France. River Research and Applications 18: 239-247. https://doi.org/10.1002/rra.667

Pywell R. F., Meek W. R., Webb N. R., Putwain P. D. \& BuLLOCK J. M. 2011. - Long-term heathland restoration on former grassland: the results of a 17-year experiment. Biological Conservation 144: 1602-1609. https://doi.org/10.1016/j.biocon.2011.02.010

Pywell R. F., Bullock J. M., Hopkins A., Walker K. J., Sparks T. H., Burke M. J. W. \& Peel S. 2002. - Restoration of species-rich grassland on arable land: assessing the limiting processes using a multi-site experiment. Journal of Applied Ecology 39: 294-309. https://doi.org/10.1046/j.13652664.2002.00718.x

R CORE TEAM 2020. - R: a language and environment for statistical computing. R Foundation for Statistical Computing, Vienna. https://www.R-project.org/, dernière consultation le 8 février 2020.

SCOTTON M. 2016. — Establishing a semi-natural grassland: effects of harvesting time and sowing density on species composition and structure of a restored Arrhenatherum elatius meadow. Agriculture, Ecosystems \& Environment 220: 35-44. https://doi. org/10.1016/j.agee.2015.12.029

SiLVERTOWN J. W. 1981. - Micro-spatial heterogeneity and seedling demography in species-rich grassland. New Phytologist 88: 117-128. https://doi.org/10.1111/j.1469-8137.1981.tb04575.x

Sparks R. E., Bayley P. B., Kohler S. L. \& Osborne L. L. 1990. Disturbance and recovery of large floodplain rivers. Environmental Management 14: 699-709. https://doi.org/10.1007/BF02394719

STAMPFLI A. 1991. - Accurate determination of vegetational change in meadows by successive point quadrat analysis. Vegetatio 96: 
185-194. https://doi.org/10.1007/BF00044979

Sullivan E., Hall N. \& Ashton P. 2020. — Restoration of upland hay meadows over an 11-year chronosequence: an evaluation of the success of green hay transfer. Restoration Ecology 28: 127-137. https://doi.org/10.1111/rec.13063

TÖröK P., Vida E., DeÁK B., Lengyel S. \& Tóthmérész B. 2011. - Grassland restoration on former croplands in Europe: an assessment of applicability of techniques and costs. Biodiversity and Conservation 20: 2311-2332. https://doi.org/10.1007/ s10531-011-9992-4

Tscharntke T., Klein A. M., Kruess A., Steffan-Dewenter I. \& THIEs C. 2005. — Landscape perspectives on agricultural intensification and biodiversity - ecosystem service management. Ecology Letters 8: 857-874. https://doi.org/10.1111/j.14610248.2005.00782.x
Wiскнам H. 2016. — ggplot2: Elegant Graphics for Data Analysis. Springer-Verlag, New York, $182 \mathrm{p}$.

WinkeL V. K. \& Roundy B. A. 1991. - Effects of cattle trampling and mechanical seedbed preparation on grass seedling emergence. Journal of Range Management 44: 176-180. https:// doi.org/10.2307/4002318

Woodcock B. A., Mcdonald A. W. \& Pywell R. F. 2011. Can long-term floodplain meadow recreation replicate species composition and functional characteristics of target grasslands? Journal of Applied Ecology 48: 1070-1078. https://doi.org/10.1111/ j.1365-2664.2011.02029.x

Young T. P., Petersen D. A. \& Clary J. J. 2005. — The ecology of restoration: historical links, emerging issues and unexplored realms. Ecology Letters 8: 662-673. https://doi.org/10.1111/j.14610248.2005.00764.x 
ANNEXES

ANNEXE 1. - Tableau de synthèse des 108 taxons après traitement de la base de données brutes, le groupe auquel ils ont été assignés et s'ils ont été conservés ou non dans les deux analyses multivariées (NMDS). Abréviation: NA, Non attribué.

\begin{tabular}{|c|c|c|c|c|c|c|c|c|}
\hline \multicolumn{2}{|r|}{ Nomenclature } & \multicolumn{2}{|c|}{ Occurrences } & \multicolumn{3}{|c|}{ Informations } & \multicolumn{2}{|c|}{ Utilisation NMDS } \\
\hline Code & Signification & $\begin{array}{l}\text { Prairie } \\
\text { source }\end{array}$ & $\begin{array}{l}\text { Placettes } \\
\text { expérimentales }\end{array}$ & Groupe & Singleton & $\begin{array}{l}\text { Indéter- } \\
\text { n minée }\end{array}$ & $\begin{array}{l}\text { Première } \\
\text { NMDS }\end{array}$ & $\begin{array}{l}\text { Deuxième } \\
\text { NMDS }\end{array}$ \\
\hline AGRSP & $\begin{array}{l}\text { Agrostis sp. (A. stolonifera L., A. canina L., } \\
\text { A. capillaris L.) }\end{array}$ & 1 & 1 & Rsp & 0 & 0 & Oui & Oui \\
\hline ALLSPH & Allium sphaerocephalon L. & 1 & 0 & Rsp & 1 & 0 & - & - \\
\hline ALOPRA & Alopecurus pratensis L. & 0 & 1 & Osp & 0 & 0 & Oui & Oui \\
\hline ALTOFF & Althaea officinalis L. & 0 & 1 & Osp & 0 & 0 & Oui & Oui \\
\hline ANALAX & Anacamptis laxiflora Lam. & 1 & 0 & Rsp & 0 & 0 & - & Oui \\
\hline ANISTE & Anisantha sterilis L. & 0 & 1 & Osp & 0 & 0 & Oui & Oui \\
\hline ANTODO & Anthoxantum odoratum L. & 1 & 1 & Rsp & 0 & 0 & Oui & Oui \\
\hline ARCLAP & Arctium lappa L. & 0 & 1 & Osp & 1 & 0 & - & - \\
\hline ARRELA & Arrhenatherum elatius L. & 1 & 1 & Rsp & 0 & 0 & Oui & Oui \\
\hline BELPER & Bellis perennis L. & 0 & 1 & Osp & 1 & 0 & - & - \\
\hline BROSP & $\begin{array}{l}\text { Bromus sp. (B. commutatus Schrad, B. } \\
\text { hordeaceus L., B. racemosus L.) }\end{array}$ & 1 & 1 & Rsp & 0 & 0 & Oui & Oui \\
\hline CARDSP & Carduus L. sp. & 0 & 1 & Osp & 1 & 0 & - & - \\
\hline CARHIR & Carex hirta L. & 1 & 0 & Rsp & 0 & 0 & - & Oui \\
\hline CARSP & $\begin{array}{l}\text { Carex sp. (hors C. flacca Schreb, C. hirta L., } \\
\text { C. otrubae Podp. et C. cf riparia) }\end{array}$ & 1 & 0 & Rsp & 0 & 0 & - & Oui \\
\hline CENDEC & Centaurea decipiens Thuill. & 1 & 1 & Rsp & 0 & 0 & Oui & Oui \\
\hline CERGLO & Cerastium glomeratum Thuill. & 0 & 1 & Osp & 0 & 0 & Oui & Oui \\
\hline CIRSP & Cirsium sp. (C. arvense L., C. vulgare Ten.) & 0 & 1 & Osp & 0 & 0 & Oui & Oui \\
\hline CONARV & Convolvulus arvensis L. & 1 & 1 & Rsp & 0 & 0 & Oui & Oui \\
\hline CONSEP & Convolvulus sepium L. & 1 & 0 & Rsp & 0 & 0 & - & - \\
\hline CRAMON & Crataegus monogyna Jacq. & 0 & 1 & Osp & 1 & 0 & - & - \\
\hline CYNCRI & Cynosurus cristatus L. & 1 & 1 & Rsp & 0 & 0 & Oui & Oui \\
\hline CYNDAC & Cynodon dactylon L. & 1 & 0 & Rsp & 0 & 0 & - & Oui \\
\hline DACGLO & Dactylis glomerata L. & 1 & 1 & Isp & 0 & 0 & Oui & Oui \\
\hline DAUCAR & Daucus carota L. & 0 & 1 & Osp & 1 & 0 & - & - \\
\hline DIPFUL & Dipsacus fullonum L. & 0 & 1 & Osp & 0 & 0 & Oui & Oui \\
\hline ELYREP & Elytrigia repens L. & 0 & 1 & Osp & 0 & 0 & Oui & Oui \\
\hline EPISP & $\begin{array}{l}\text { Epilobium sp (E. hirsutum L., E. tetragonum } \\
\text { L.) }\end{array}$ & 0 & 1 & Osp & 0 & 0 & Oui & Oui \\
\hline EQUARV & Equisetum arvense $\mathrm{L}$. & 0 & 1 & Osp & 0 & 0 & Oui & Oui \\
\hline ERISP & Erigeron L. sp. & 0 & 1 & Osp & 0 & 0 & Oui & Oui \\
\hline FESRUB & Festuca L. gr. rubra & 1 & 0 & Rsp & 0 & 0 & - & Oui \\
\hline FRAEXC & Fraxinus excelsior $\mathrm{L}$. & 0 & 1 & Osp & 0 & 0 & Oui & Oui \\
\hline GALAPA & Galium aparine L. & 0 & 1 & Osp & 0 & 0 & Oui & Oui \\
\hline GALSP & Galium sp. (dont G. mollugo L., G. verum L.) & 1 & 1 & Rsp & 0 & 0 & - & - \\
\hline GAUFRA & Gaudinia fragilis Beauv. & 1 & 1 & Rsp & 0 & 0 & Oui & Oui \\
\hline GERDIS & Geranium dissectum L. & 1 & 1 & Rsp & 0 & 0 & Oui & Oui \\
\hline HEDHEL & Hedera helix L. & 0 & 1 & Osp & 0 & 0 & Oui & Oui \\
\hline HELECH & Helminthotheca echioides L. & 0 & 1 & Osp & 0 & 0 & Oui & Oui \\
\hline HOLLAN & Holcus lanatus L. & 1 & 1 & Rsp & 0 & 0 & Oui & Oui \\
\hline HORMUR & Hordeum murinum L. & 0 & 1 & Osp & 1 & 0 & - & - \\
\hline HORSEC & Hordeum secalinum Schreb. & 1 & 1 & Rsp & 0 & 0 & Oui & Oui \\
\hline HYPRAD & Hypochaeris radicata $\mathrm{L}$. & 0 & 1 & Osp & 0 & 0 & Oui & Oui \\
\hline INDET01 & NA & 0 & 1 & Osp & 1 & 1 & - & - \\
\hline INDET02 & Apiaceae non determinees & 0 & 1 & Osp & 0 & 1 & - & - \\
\hline INDET03 & Asteraceae non determinees & 0 & 1 & Osp & 0 & 1 & - & - \\
\hline INDET04 & cf Acer negundo L. & 0 & 1 & Osp & 1 & 1 & - & - \\
\hline INDET05 & cf Fraxinus excelsior L. & 1 & 0 & Rsp & 1 & 1 & - & - \\
\hline INDET07 & cf Lotus L. sp. & 0 & 1 & Osp & 1 & 1 & - & - \\
\hline INDET08 & cf Lythrum salicaria L. & 0 & 1 & Osp & 1 & 1 & - & - \\
\hline INDET09 & cf Picris hieracioides L. & 0 & 1 & Osp & 1 & 1 & - & - \\
\hline INDET10 & cf Xanthium L. sp & 0 & 1 & Osp & 1 & 1 & - & - \\
\hline INDET11 & NA & 0 & 1 & Osp & 1 & 1 & - & - \\
\hline INDET13 & NA & 0 & 1 & Osp & 1 & 1 & - & - \\
\hline INDET14 & NA & 0 & 1 & Osp & 1 & 1 & - & - \\
\hline INDET15 & cf Polygonum L. sp & 0 & 1 & Osp & 0 & 0 & - & - \\
\hline JUNSP & Juncus sp. (dont $J$. acutiflorus Hoffm.) & 1 & 0 & Rsp & 0 & 0 & - & - \\
\hline LAPCOM & Lapsana communis L. & 0 & 1 & Osp & 0 & 0 & Oui & Oui \\
\hline LATNIS & Lathyrus nissolia L. & 1 & 1 & Rsp & 0 & 0 & Oui & Oui \\
\hline LATPRA & Lathyrus pratense L. & 1 & 1 & Rsp & 0 & 0 & Oui & Oui \\
\hline LEUVUL & Leucanthemum vulgare Lam. & 0 & 1 & Osp & 1 & 0 & - & - \\
\hline LINUSI & Linum usitatissimum L. & 1 & 1 & Rsp & 0 & 0 & Oui & Oui \\
\hline
\end{tabular}


ANNEXE 1. - Suite.

\begin{tabular}{|c|c|c|c|c|c|c|c|c|}
\hline \multicolumn{2}{|r|}{ Nomenclature } & \multicolumn{2}{|c|}{ Occurrences } & \multicolumn{3}{|c|}{ Informations } & \multicolumn{2}{|c|}{ Utilisation NMDS } \\
\hline Code & Signification & $\begin{array}{l}\text { Prairie } \\
\text { source }\end{array}$ & $\begin{array}{l}\text { Placettes } \\
\text { expérimentales }\end{array}$ & Groupe & Singleton & $\begin{array}{l}\text { Indéter- } \\
\text { minée }\end{array}$ & $\begin{array}{l}\text { Première } \\
\text { NMDS }\end{array}$ & $\begin{array}{l}\text { Deuxième } \\
\text { NMDS }\end{array}$ \\
\hline LOLPER & Lolium perenne L. & 1 & 1 & Isp & 0 & 0 & Oui & Oui \\
\hline LOLSCH & Lolium x Schedonorus & 0 & 1 & Osp & 0 & 0 & Oui & Oui \\
\hline LOTCOR & Lotus corniculatus L. & 1 & 1 & Isp & 0 & 0 & Oui & Oui \\
\hline LOTGLA & Lotus glaber Mill. & 1 & 1 & Rsp & 0 & 0 & Oui & Oui \\
\hline LOTPED & Lotus pedunculatus Cav. & 1 & 1 & Rsp & 0 & 0 & Oui & Oui \\
\hline LYSARV & Lysimachia arvensis L. & 0 & 1 & Osp & 0 & 0 & Oui & Oui \\
\hline LYSNUM & Lysimachia nummularia L. & 0 & 1 & Osp & 1 & 0 & - & - \\
\hline MEDLUP & Medicago Iupulina L. & 0 & 1 & Osp & 0 & 0 & Oui & Oui \\
\hline MEDSAT & Medicago sativa L. & 0 & 1 & Osp & 0 & 0 & Oui & Oui \\
\hline MEDSP & $\begin{array}{l}\text { Medicago arabica Huds.et/ou Medicago } \\
\text { polymorpha L. }\end{array}$ & 0 & 1 & Osp & 0 & 0 & Oui & Oui \\
\hline MYOSP & Myosotis L. sp. & 0 & 1 & Osp & 0 & 0 & Oui & Oui \\
\hline OENPIM & Oenanthe pimpineloides L. & 1 & 1 & Rsp & 0 & 0 & Oui & Oui \\
\hline OPHSP & Ophrys apifera, Ophrys sp. & 0 & 1 & Osp & 0 & 0 & Oui & Oui \\
\hline PLALAN & Plantago lanceolata L. & 1 & 1 & Rsp & 0 & 0 & Oui & Oui \\
\hline PLAMAJ & Plantago major L. & 0 & 1 & Osp & 1 & 0 & - & - \\
\hline POAAN & Poa annua L. & 0 & 1 & Osp & 0 & 0 & Oui & Oui \\
\hline POAPRA & Poa pratensis L. & 1 & 0 & Rsp & 0 & 0 & - & Oui \\
\hline POASP & Poa L. sp. & 0 & 1 & Osp & 1 & 0 & - & - \\
\hline POATRI & Poa trivialis $\mathrm{L}$. & 1 & 1 & Rsp & 0 & 0 & Oui & Oui \\
\hline POTREP & Potentilla reptans L. & 1 & 1 & Rsp & 0 & 0 & Oui & Oui \\
\hline POTSP & Potentilla L. sp. & 0 & 1 & Osp & 1 & 0 & - & - \\
\hline PRAAUS & Phragmites australis Steud. & 0 & 1 & Osp & 0 & 0 & Oui & Oui \\
\hline PRUSP & Prunus L. sp. & 0 & 1 & Osp & 0 & 0 & Oui & Oui \\
\hline PRUVUL & Prunella vulgaris L. & 1 & 1 & Rsp & 0 & 0 & Oui & Oui \\
\hline RANACR & Ranunculus acris L. & 1 & 1 & Rsp & 0 & 0 & Oui & Oui \\
\hline RANSP & Ranunculus repens L. (hors $R$. acris) & 1 & 1 & Rsp & 0 & 0 & Oui & Oui \\
\hline RAPRAP & Raphanus raphanistrum $\mathrm{L}$. & 0 & 1 & Osp & 0 & 0 & Oui & Oui \\
\hline RHIANG & Rhinanthus angustifolius C.C.Gmel. & 1 & 0 & Rsp & 0 & 0 & - & Oui \\
\hline RUBSP & Rubus L. sp. & 0 & 1 & Osp & 0 & 0 & Oui & Oui \\
\hline RUMACE & Rumex acetosa L. & 1 & 1 & Rsp & 0 & 0 & Oui & Oui \\
\hline RUMSP & $\begin{array}{l}\text { Rumex sp ( } R \text {. crispus L., } R \text {. conglomeratus } \\
\quad \text { Murray) }\end{array}$ & 1 & 1 & Rsp & 0 & 0 & Oui & Oui \\
\hline SCHARU & Schedonorus arundinaceus Schreb. & 1 & 1 & Isp & 0 & 0 & Oui & Oui \\
\hline SONSP & Sonchus asper L. / Sonchus oleraceus L. & 0 & 1 & Osp & 0 & 0 & Oui & Oui \\
\hline SORHAL & cf Sorghum halepense L. & 0 & 1 & Osp & 0 & 0 & Oui & Oui \\
\hline STEGRA & Stellaria graminea L. & 1 & 0 & Rsp & 0 & 0 & - & Oui \\
\hline TAROFF & Taraxacum officinale H. Wigg. s.I. & 1 & 1 & Rsp & 0 & 0 & Oui & Oui \\
\hline TRICAM & Trifolium campestre Schreb. & 0 & 1 & Osp & 1 & 0 & - & - \\
\hline TRIcfPRA & Trifolium cf pratense L. & 0 & 1 & Osp & 1 & 0 & - & - \\
\hline TRIDUB & Trifolium dubium Sibth. & 0 & 1 & Osp & 0 & 0 & Oui & Oui \\
\hline TRIFLA & Trisetum flavescens Beauv. & 0 & 1 & Osp & 0 & 0 & Oui & Oui \\
\hline TRIHYB & Trifolium hybridum L. & 0 & 1 & Isp & 0 & 0 & Oui & Oui \\
\hline TRIPAT & Trifolium patens Schreb. & 1 & 1 & Rsp & 0 & 0 & Oui & Oui \\
\hline TRIPRA & Trifolium pratense L. & 1 & 1 & Rsp & 0 & 0 & Oui & Oui \\
\hline TRIREP & Trifolium repens L. & 1 & 1 & Isp & 0 & 0 & Oui & Oui \\
\hline VALLOC & Valerianella locusta L. & 0 & 1 & Osp & 0 & 0 & Oui & Oui \\
\hline VEROFF & Verbena officinalis L. & 0 & 1 & Osp & 0 & 0 & Oui & Oui \\
\hline VERSP & Veronica L. sp & 0 & 1 & Osp & 0 & 0 & Oui & Oui \\
\hline VICSP & Ervum tetraspermum L., Vicia L. sp. & 0 & 1 & Osp & 0 & 0 & Oui & Oui \\
\hline
\end{tabular}


ANNEXE 2. - Ordination de type NMDS (Non Metric Multidimensional Scaling) sur la matrice [120 × 70] des relevés floristiques réalisés sur les placettes. A, Plan factoriel NMDS1 - NMDS2 des espèces (voir Annexe 1 pour la correspondance entre les codes et les noms d'espèces) expérimentales en 2015,2017 et 2019; B, nombre (pourcentage) des espèces appartenant aux trois groupes (semis/référence/autres) projetées en partie positive et négative des axes NMDS1 et NMDS2.

A

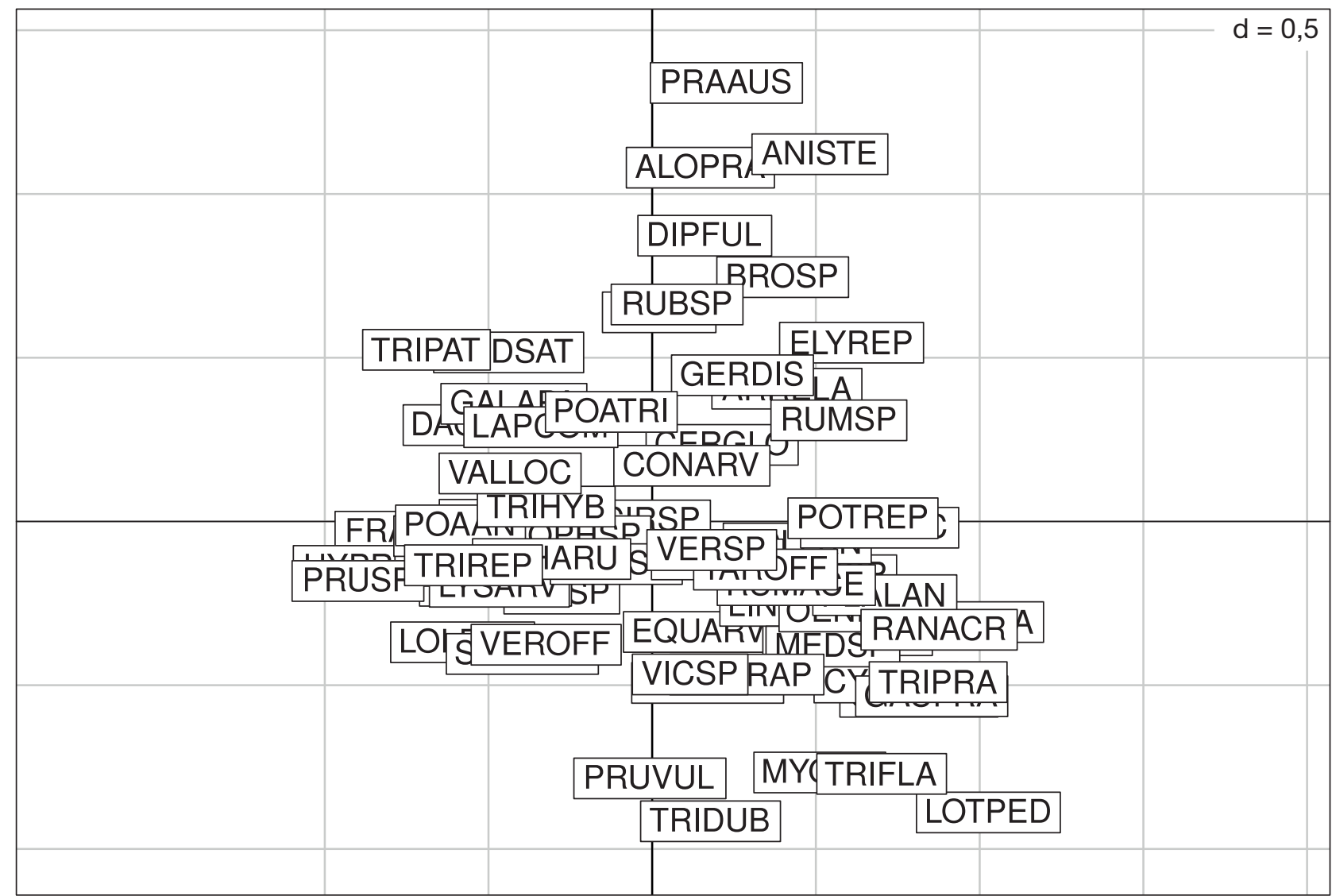

B

\begin{tabular}{|c|c|c|c|c|c|}
\hline & \multirow[b]{2}{*}{ Total } & \multicolumn{2}{|c|}{ NMDS1 } & \multicolumn{2}{|c|}{ NMDS2 } \\
\hline & & Négatif & Positif & Négatif & Positif \\
\hline Espèces du semis initial (Isp) & $6(100 \%)$ & $6(100 \%)$ & $0(0 \%)$ & $4(67 \%)$ & $3(33 \%)$ \\
\hline Espèces de référence (Rsp) & $28(100 \%)$ & $5(18 \%)$ & $23(82 \%)$ & $20(71 \%)$ & $8(29 \%)$ \\
\hline Autres espèces (Osp) & $36(100 \%)$ & $18(50 \%)$ & $18(50 \%)$ & $22(61 \%)$ & $14(39 \%)$ \\
\hline
\end{tabular}


ANNEXE 3. - Ordination de type NMDS (Non Metric Multidimensional Scaling) sur la matrice [132 × 78] des relevés floristiques réalisés sur les placettes expérimentales en 2015, 2017 et 2019 et dans la prairie donneuse en 2013 et en 2017. A, Plan factoriel NMDS1 - NMDS2 des espèces (voir Annexe 1 pour la correspondance entre les codes et les noms d'espèces); $\mathbf{B}$, nombre (pourcentage) des espèces appartenant aux trois groupes (semis/référence/autres) projetées en partie positive et négative des axes NMDS1 et NMDS2.

A

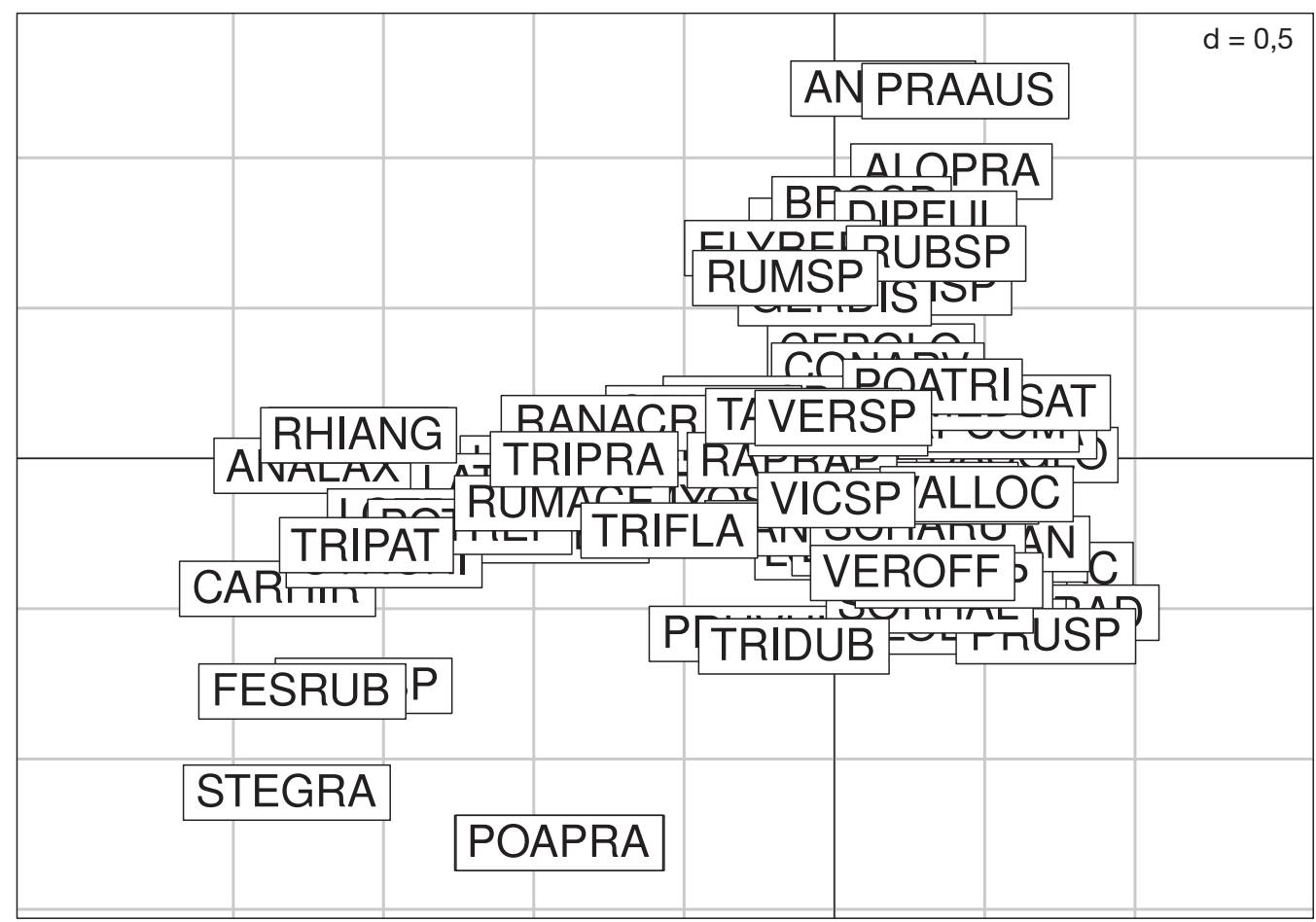

B

\begin{tabular}{|c|c|c|c|c|c|}
\hline & \multirow[b]{2}{*}{ Total } & \multicolumn{2}{|c|}{ NMDS1 } & \multicolumn{2}{|c|}{ NMDS2 } \\
\hline & & Négatif & Positif & Négatif & Positif \\
\hline $\begin{array}{l}\text { Espèces du semis initial (Isp) } \\
\text { Espèces de référence (Rsp) } \\
\text { Autres espèces (Osp) }\end{array}$ & $\begin{array}{r}6(100 \%) \\
28(100 \%) \\
36(100 \%)\end{array}$ & $\begin{array}{c}6(100 \%) \\
5(18 \%) \\
18(50 \%)\end{array}$ & $\begin{array}{c}0(0 \%) \\
23(82 \%) \\
18(50 \%)\end{array}$ & $\begin{array}{r}4(67 \%) \\
20(71 \%) \\
22(61 \%)\end{array}$ & $\begin{array}{r}3(33 \%) \\
8(29 \%) \\
14(39 \%)\end{array}$ \\
\hline
\end{tabular}

